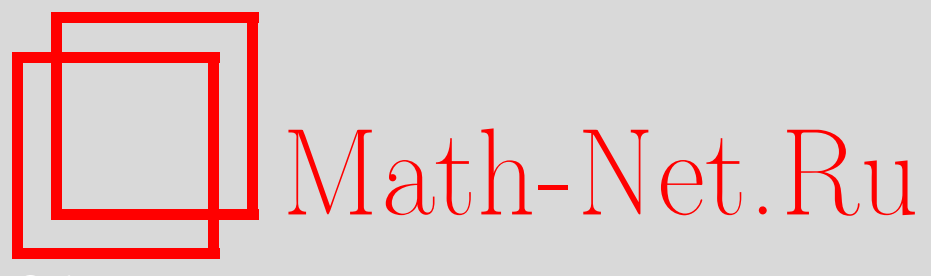

В. И. Кукулин, В. Н. Померанцев, О. А. Рубцова, Метод пакетной дискретизации континуума для решения трехчастичной задачи рассеяния, ТМФ, 2007, том 150, номер 3, 473-497

DOI: https://doi.org/10.4213/tmf5991

Использование Общероссийского математического портала Math-Net.Ru подразумевает, что вы прочитали и согласны с пользовательским соглашением http://www . mathnet.ru/rus/agreement

Параметры загрузки:

IP : 54.210 .77 .194

26 апреля 2023 г., 15:21:58

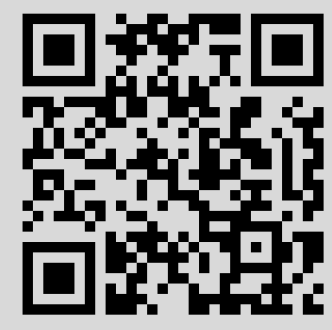




\title{
МЕТОД ПАКЕТНОЙ ДИСКРЕТИЗАЦИИ КОНТИНУУМА ДЛЯ РЕШЕНИЯ ТРЕХЧАСТИЧНОЙ ЗАДАЧИ РАССЕЯНИЯ
}

\begin{abstract}
Развитый ранее метод пакетной дискретизации континуума в задаче рассеяния обобщен на систему трех частиц. Для каждого асимптотического канала построен базис трехчастичных волновых пакетов, являющихся квадратичноинтегрируемыми функциями. Показано, что проекции канальных резольвент на подпространство трехчастичных волновых пакетов определяются диагональными матрицами, для собственных значений которых приведены явные формулы. Амплитуды процессов $2 \rightarrow 2$ выражены явно через "пакетные" конечномерные проекции полной резольвенты. В качестве иллюстрации развитого формализма рассчитаны дифференциальное сечение упругого рассеяния дейтрона на тяжелом ядре выше трехчастичного порога развала и $s$-волновая квартетная амплитуда $(n-d)$-рассеяния. Результаты расчетов хорошо согласуются с результатами других методов. Предлагаемая схема решения трехчастичной задачи рассеяния сопоставима по сложности с решением аналогичной задачи для связанных состояний.
\end{abstract}

Ключевые слова: квантовая теория рассеяния, малочастичные системы, дискретизация континуума.

\section{1. ВВЕДЕНИЕ}

В последние десятилетия основной прогресс в решении многочастичных квантовых задач рассеяния достигнут с помощью метода интегральных и интегродифференциальных уравнений Фаддеева-Якубовского, которые были получены и математически обоснованы в 60-70 гг. прошлого века [1], [2]. С тех пор методы численного решения этих уравнений постоянно совершенствовались, что позволило уже к середине 80-х гг. выполнить точные расчеты трех- и, частично, четырехнуклонных задач с реалистическими $N N$ - и $3 N$-взаимодействиями [3]-[6]. Однако до сих пор практическое решение таких задач связано с огромным объемом вычислений и поэтому требует применения наиболее мощных из имеющихся суперкомпьютеров [4]. Но даже самая современная вычислительная техника не в состоянии преодолеть все численные проблемы, возникающие в рамках традиционных подходов. Например,

*Научно-исследовательский институт ядерной физики им. Д. В. Скобельцына, Московский государственный университет, Москва, Россия. E-mail: kukulin@nucl-th.sinp.msu.ru, pomeran@nucl-th.sinp.msu.ru,rubtsova@nucl-th.sinp.msu.ru; rubtsova-olga@yandex.ru 
при решении четырехнуклонной задачи очень трудно продвинуться в область выше порога трехчастичного развала [5]. Более того, даже при решении трехнуклонной задачи $(N+d)$-рассеяния на основе традиционных подходов с большим трудом удается описать область энергий выше 200 МэВ из-за огромного числа участвующих парциальных волн. Поэтому приходится отказаться от парциально-волнового разложения и перейти к решению уравнений Фаддеева непосредственно в шестимерном пространстве координат Якоби [6]. Трудности усугубляются, если необходимо учитывать дальнодействующее кулоновское взаимодействие и трехчастичные силы, особенно если они зависят от спина. Поэтому даже весьма быстрый прогресс в создании мощных суперкомпьютеров, наблюдаемый в последние годы, не позволяет в настоящий момент преодолеть все сложности, возникающие при решении многочастичных задач рассеяния на основе точных интегральных или интегродифференциальных уравнений.

С другой стороны, в последние годы было развито много весьма перспективных методов решения многочастичных задач для дискретного спектра. В частности, были выполнены реалистичные расчеты для дискретного и квазидискретного спектров систем с пятью, шестью и бо́льшим количеством нуклонов (или других фермионов). Для бозонных систем в настоящее время существуют методы (типа метода Монте-Карло), позволяющие точно рассчитывать состояния систем из сотен или даже тысяч взаимодействующих частиц. Таким образом, существует разительный контраст: трудоемкости расчетов одной и той же физической системы (с общим гамильтонианом) при разных граничных условиях на волновые функции различаются в сотни и тысячи раз. Это представляется весьма странным, и возникает желание изменить весь подход к практическому решению задач рассеяния, сблизив методы решения задач для непрерывного и дискретного спектров. Естественным путем к этому является дискретизация непрерывного спектра.

Необходимо отметить, что идея дискретизации непрерывного спектра при решении задач рассеяния возникла давно и активно используется до сих пор. Напрямую или косвенно она применяется в различных методах, основанных на разложении волновых функций рассеяния по $L_{2}$-базисам $\left(L_{2}\right.$-дискретизация). Наиболее известные из них - $J$-матричный подход [7], $R$-матричный метод [8], а также различные варианты метода связанных каналов дискретизованного континуума (мы будем далее называть его CDCC-методом, используя аббревиатуру, отвечающую английскому термину continuum discretized coupled channel method) [9]-[11], который был разработан для решения задач рассеяния составных частиц на средних и тяжелых ядрах. В CDCC-методе дискретизация континуума проводится только для гамильтониана двухчастичной подсистемы, включающей падающую составную частицу. Это позволяет получить из уравнения Шредингера для полной волновой функции систему одномерных интегродифференциальных уравнений метода связанных каналов. Поскольку упомянутые выше методы используют уравнения Шредингера, для нахождения наблюдаемых необходимо проводить процедуру сшивки приближенных решений во внутренней области с асимптотическими решениями во внешней области.

Однако используемая в традиционном CDCC-методе процедура сшивки волновой функции, описывающей движение центра масс составной частицы, которая взаимодействует с мишенью в своих возбужденных состояниях, с асимптотическими 
решениями, описывающими движение лишь центра масс, не является бесспорной. В самом деле, для возбужденных состояний составной частицы речь идет по существу о процессах ее развала в поле тяжелого ядра, а правильные граничные условия для волновой функции трехчастичного развала не могут быть учтены лишь с помощью указанной выше сшивки (заметим, что в середине 80-х гг. CDCC-метод был подвергнут жесткой критике именно по этой причине.) Поэтому в нашем подходе мы попытались отказаться от этой не вполне корректной процедуры сшивки и использовали вместо этого технику волновых пакетов по переменной относительного движения частицы-снаряда и мишени.

С другой стороны, существуют несколько других методов решения задач рассеяния в $L_{2}$-базисах. Эти методы основаны на интегральных уравнениях теории рассеяния и позволяют избежать явной процедуры сшивки решений. $\mathrm{K}$ ним относятся метод моментов [12], методы, основанные на вариационном принципе Швингера [13], метод интегральных преобразований Лоренца [14]. Недавно появилась серия работ [15], в которых авторы развивают чисто алгебраический подход к решению интегральных уравнений Меркурьева-Фаддеева для трехчастичной системы с кулоновским взаимодействием. При этом используется аппроксимация ядер этих интегральных уравнений операторами конечного ранга, что приводит к алгебраической системе уравнений.

Предлагаемый нами пакетный подход фактически объединяет идеи последовательной дискретизации непрерывного спектра и использования интегральной формулировки теории рассеяния. Как было показано в наших предыдущих работах [16], [17], дискретизация континуума с помощью волновых пакетов в двухчастичной задаче приводит к конечномерному ядру в уравнении Липпмана-Швингера, т.е. к сведе́нию этого интегрального уравнения к алгебраическому. Характерной особенностью проектора, построенного из волновых пакетов, является то, что матрица, отвечающая проекции свободной резольвенты, оказывается диагональной, и ее матричные элементы имеют простой вид. При переходе к трехчастичной системе мы строим трехчастичные волновые пакеты, которые снова являются квадратичноинтегрируемыми в полном трехчастичном пространстве, т.е. принадлежат гильбертову пространству системы трех тел. Свойства построенных трехчастичных базисов позволяют получить явные конечномерные диагональные представления теперь уже для трехчастичных резольвент каналов. Далее эти удобные представления можно использовать в ядрах интегральных уравнений для нахождения наблюдаемых.

В наших предыдущих работах [16], [17] было показано, что пакетные состояния можно аппроксимировать (с любой точностью) так называемыми псевдосостояниями, получаемыми при диагонализации соответствующего гамильтониана в конечном $L_{2}$-базисе, например, осцилляторном или гауссовом базисах [17]. Таким образом, пакетный метод приводит к формулировке теории рассеяния целиком в терминах ортонормированных функций из $L_{2}$, т.е. на том языке, который используется при описании дискретного спектра. Применение осцилляторного или гауссова базиса для аппроксимации пакетов особенно важно для многочастичных задач. Здесь появляется возможность использовать все те преимущества многочастичного аналитического базиса, которые уже давно и с большим успехом используются при анализе дискретного спектра в системах нескольких или многих тел: например, полностью аналитическую перевязку потенциалов взаимодействия или парных операторов рас- 
сеяния при переходе от одного набора координат Якоби к другому, простоту перехода от координатного представления к импульсному и т.д. Поэтому можно полагать, что решение многочастичных квантовых задач рассеяния на основе предлагаемого формализма волновых пакетов окажется ненамного сложнее решения задач для дискретного спектра и будет доступно обычным современным персональным компьютерам.

Настоящая работа имеет следующую структуру. В разделе 2 проводится обобщение пакетного формализма на случай трех частиц. Изучаются свойства трехчастичных волновых пакетов. Выводятся конечномерные аналитические представления для канальных резольвент. Раздел 3 посвящен применению развитого формализма для решения задачи рассеяния составной частицы на тяжелом ядре. В разделе 4 рассматривается решение задачи рассеяния трех тождественных частиц. В заключительном разделе 5 приведены основные выводы.

\section{2. ТРЕХЧАСТИЧНЫЕ ВОЛНОВЫЕ ПАКЕТЫ И ИХ СВОЙСТВА}

2.1. Постановка трехчастичной задачи. Рассмотрим общую задачу рассеяния в системе трех бесспиновых частиц с короткодействующими парными силами. Гамильтониан системы имеет вид

$$
H=H_{0}+V_{1}+V_{2}+V_{3}
$$

Здесь $H_{0}$ - оператор кинетической энергии всей системы (в системе центра масс), а $V_{a}$ - операторы взаимодействия частиц $b$ и $c(a, b, c$ принимают попарно различные значения из множества $\{1,2,3\})$.

Будем далее рассматривать процессы, в начальном и конечном состояниях которых две частицы связаны, а третья свободна, т.е. процессы типа $2 \rightarrow 2$. Асимптотическим состояниям для таких процессов отвечают гамильтонианы каналов $H_{a}$,

$$
H_{a}=H_{0}+V_{a}, \quad a=1,2,3 .
$$

Полный гамильтониан системы записывается через канальные как

$$
H=H_{a}+U_{a}, \quad a=1,2,3,
$$

где $U_{a}=V_{b}+V_{c}$ - так называемое внешнее взаимодействие в канале $a$.

Начальное состояние задается собственной функцией гамильтониана $H_{a}$

$$
\left|\Psi_{a n}(E)\right\rangle=\left|\hat{\psi}_{n}\left(\varepsilon_{n}^{a}\right), \psi_{0}^{a}\left(E-\varepsilon_{n}^{a}\right)\right\rangle,
$$

которая является произведением функции связанного состояния $\left|\hat{\psi}_{n}\left(\varepsilon_{n}^{a}\right)\right\rangle$ гамильтониана подсистемы $\{b c\}$ с энергией $\varepsilon_{n}^{a}$ и функции $\left|\psi_{0}^{a}\left(E-\varepsilon_{n}^{a}\right)\right\rangle$, описывающей свободное движение частицы $a$ относительно пары $\{b c\}$. Конечное состояние, в котором имеется связанная пара $\{a c\}$ и свободная частица $b$, описывается волновой функцией

$$
\left|\Psi_{b m}(E)\right\rangle=\left|\hat{\psi}_{m}\left(\varepsilon_{m}^{b}\right), \psi_{0}^{b}\left(E-\varepsilon_{m}^{b}\right)\right\rangle .
$$

Амплитуда перехода для процесса $a \rightarrow b$ определяется как матричный элемент оператора перехода

$$
\mathcal{T}_{a b}=U_{b}+U_{b} G(E) U_{a}
$$


между этими состояниями:

$$
A_{b a}(E)=\left\langle\Psi_{b m}(E)\left|U_{b}+U_{b} G(E) U_{a}\right| \Psi_{a n}(E)\right\rangle,
$$

где $G(\zeta)=[\zeta-H]^{-1}$ - резольвента полного гамильтониана (1). В случае короткодействующих парных сил функции $\left\langle\Psi_{b m}(E)\right| U_{b}$ и $U_{a}\left|\Psi_{a n}(E)\right\rangle$ в “обкладках" полной резольвенты в выражении (7) представляют собой результат действия внешних потенциалов $U$ на волновые функции начального и конечного состояний, и поэтому они, как легко видеть, являются квадратично-интегрируемыми по всем пространственным переменным. Следовательно, ядро резольвенты $G(E)$ в (7) эффективно “обрезается" по всем координатам конфигурационного пространства. Таким образом, для нахождения амплитуд перехода, как и в двухчастичной задаче (см. [16], [17]), достаточно знать ядро резольвенты лишь во внутренней области этого пространства. Это означает, что можно разложить ядро резольвенты по некоторому полному (трехчастичному) базису квадратично-интегрируемых функций и в расчетах оставить конечное число членов такого разложения. Для этих целей будем использовать технику стационарных волновых пакетов, предложенную в наших предыдущих работах [16], [17].

Пакетный базис строится из стационарных волновых пакетов, которые получаются из волновых функций непрерывного спектра гамильтониана путем интегрирования по конечному интервалу спектра. Для трехчастичной системы удобно ввести три пакетных базиса для трех каналов, отвечающих трем возможным разбиениям вида $a+\{b c\}$ для этой системы. Так, в канале $a$ стационарные трехчастичные волновые пакеты (ТВП) соответствуют собственным функциям канального гамильтониана $H_{a}$.

Определим, как обычно [2], координаты Якоби для канала $a$ : координату относительного движения частиц $b$ и $c$ будем обозначать как $\boldsymbol{\xi}_{a}$, а координату движения третьей частицы $a$ относительно центра масс пары $\{b c\}-$ как $\boldsymbol{\eta}_{a}$. Радиус-векторы отдельных частиц будем обозначать символом $\mathbf{r}_{i}$.

Гамильтониан канала $H_{a}$ записывается в координатах Якоби как прямая сумма двухчастичных гамильтонианов:

$$
H_{a}=h_{0}^{a} \oplus h_{a},
$$

где $h_{a}$ - гамильтониан подсистемы $\{b c\}$, и $h_{0}^{a}$ - гамильтониан свободного движения частицы $a$ относительно пары $\{b c\}$. Поэтому собственные функции канального гамильтониана $H_{a}$ являются произведениями двухчастичных собственных функций гамильтонианов $h_{a}$ и $h_{0}^{a}$, а ТВП строятся из произведений двухчастичных пакетов для этих гамильтонианов. Построим сначала двухчастичные пакетные базисы для $h_{a}$ и $h_{0}^{a}$.

2.2. Пакетный базис для гамильтониана $h_{a}$. Рассмотрим спектр состояний гамильтониана двухчастичной подсистемы $h_{a}$, отвечающих определенным значениям $l_{a}$ и $m_{a}$ орбитального момента $\mathbf{l}_{a}$ и его проекции на ось $z$, соответственно. В случае короткодействующих взаимодействий оператор $h_{a}$ имеет конечный дискретный спектр $\left\{\varepsilon_{k}^{l_{a}}\right\}_{i=1}^{k_{l a}}$ и соответствующий набор собственных волновых функций $\left\{\left|\hat{\psi}_{k}^{l_{a} m_{a}}\right\rangle\right\}_{k=1}^{k_{l_{a}}}$, а также непрерывный спектр $(0, \infty)$, которому соответствуют функции $\left|\psi\left(E, l_{a} m_{a}\right)\right\rangle$ (они не являются квадратично-интегрируемыми). Поскольку 
спектр является $\left(2 l_{a}+1\right)$-кратно вырожденным по значению $m_{a}$, мы будем опускать этот индекс там, где нет необходимости. Ниже обсуждаются радиальные части волновых функций.

Дискретизацией непрерывного спектра гамильтониана $h_{a}$ назовем разбиение части непрерывного спектра на набор непересекающихся интервалов: пусть

$$
0=E_{k_{l_{a}}}^{l_{a}}<E_{k_{l_{a}}+1}^{l_{a}}<\cdots<E_{i}^{l_{a}}<\cdots<E_{n_{l_{a}}}^{l_{a}}, \quad \delta_{i}^{l_{a}}=E_{i}^{l_{a}}-E_{i-1}^{l_{a}}, \quad i=k_{l_{a}}+1, \ldots, n_{l_{a}},
$$

где $\delta_{i}^{l_{a}}$ - ширины интервалов разбиения. Такому разбиению соответствует набор стационарных волновых пакетов $\left|Z_{i}^{l_{a}}\right\rangle$, построенных из точных волновых функций $\left|\psi\left(E, l_{a}\right)\right\rangle$ непрерывного спектра гамильтониана $h_{a}$,

$$
\left|Z_{i}^{l_{a} m_{a}}\right\rangle=\frac{1}{\sqrt{\delta_{i}^{l_{a}}}} \int_{E_{i-1}^{l_{a}}}^{E_{i}^{l_{a}}} d E\left|\psi\left(E, l_{a} m_{a}\right)\right\rangle, \quad i=k_{l_{a}}+1, \ldots, n_{l_{a}} .
$$

Свойства этих пакетов подробно изучены нами ранее [16], [17]. В частности, функции $\left|Z_{i}^{l_{a}}\right\rangle$ являются квадратично-интегрируемыми и вместе с функциями связанных состояний гамильтониана $h_{a}$ образуют ортонормированный базис, в котором матрица гамильтониана диагональна и имеет простые собственные значения:

$$
\begin{gathered}
\left\langle Z_{i}^{l_{a}} \mid Z_{j}^{l_{a}}\right\rangle=\delta_{i j}, \quad\left\langle Z_{i}^{l_{a}} \mid \hat{\psi}_{k}^{l_{a}}\right\rangle=0, \\
\left\langle Z_{i}^{l_{a}}\left|h_{a}\right| Z_{j}^{l_{a}}\right\rangle=E_{i}^{* l_{a}} \delta_{i j}, \quad E_{i}^{* l_{a}}=\frac{1}{2}\left(E_{i-1}^{l_{a}}+E_{i}^{l_{a}}\right) .
\end{gathered}
$$

Собственные значения $E_{i}^{* l_{a}}$ матрицы $\left\langle Z_{i}^{l_{a}}\left|h_{a}\right| Z_{j}^{l_{a}}\right\rangle$ являются средними точками интервалов разбиения и образуют дискретизованный континуум.

Проектор на пакетное подпространство гамильтониана $h_{a}$ определяется как сумма проекторов на состояния дискретного и дискретизованного непрерывного спектров:

$$
\mathbb{I}^{l_{a} m_{a}}=\sum_{k=1}^{k_{l_{a}}}\left|\hat{\psi}_{k}^{l_{a} m_{a}}\right\rangle\left\langle\hat{\psi}_{k}^{l_{a} m_{a}}\left|+\sum_{i=k_{l_{a}}+1}^{n_{l_{a}}}\right| Z_{i}^{l_{a} m_{a}}\right\rangle\left\langle Z_{i}^{l_{a} m_{a}}\right|
$$

Для простоты будем при использовании пакетного базиса обозначать функции связанных состояний тем же символом, что и функции дискретизованного континуума:

$$
\left|\hat{\psi}_{k}^{l_{a}}\right\rangle \equiv\left|Z_{k}^{l_{a}}\right\rangle, \quad k=1, \ldots, k_{l_{a}} .
$$

Пакетный проектор (11), действуя на волновую функцию непрерывного спектра $\left|\psi\left(E, l_{a}\right)\right\rangle$, дает волновой пакет, относящийся к тому энергетическому интервалу, которому принадлежит энергия $E$ :

$$
\mathbb{I}^{l_{a} m_{a}}\left|\psi\left(E, l_{a} m_{a}\right)\right\rangle= \begin{cases}\frac{\left|Z_{i}^{l_{a}}\right\rangle}{\sqrt{\delta_{i}^{l_{a}}}}, & E \in\left(E_{i-1}^{l_{a}}, E_{i}^{l_{a}}\right), \\ 0, & E \notin\left(0, E_{n_{l_{a}}}^{l_{a}}\right) .\end{cases}
$$


Двухчастичную резольвенту $g_{a}^{(+)}(E)=\left[E+i 0-h_{a}\right]^{-1}$ гамильтониана $h_{a}$ можно записать в виде спектральной суммы,

$$
g_{a}^{(+)}(E)=\sum_{l_{a}=0}^{\infty} \sum_{m=-l_{a}}^{l_{a}}\left(\sum_{k=1}^{k_{l_{a}}} \frac{\left|\hat{\psi}_{k}^{l_{a} m_{a}}\right\rangle\left\langle\hat{\psi}_{k}^{l_{a} m_{a}}\right|}{E+i 0-\varepsilon_{i}^{l_{a}}}+\int_{0}^{\infty} d E_{1} \frac{\left|\psi\left(E_{1}, l_{a} m_{a}\right)\right\rangle\left\langle\psi\left(E_{1}, l_{a} m_{a}\right)\right|}{E+i 0-E_{1}}\right) .
$$

Действуя справа и слева на это выражение проектором $\mathbb{I}^{l_{a} m_{a}}$, получаем формулу для проекции резольвенты на пакетное подпространство:

$$
\mathbb{I}^{l_{a} m_{a}} g_{a}^{(+)}(E) \mathbb{I}^{l_{a} m_{a}}=\sum_{i=1}^{n_{l_{a}}} g_{i}^{l_{a} m_{a}}(E)\left|Z_{i}^{l_{a} m_{a}}\right\rangle\left\langle Z_{i}^{l_{a} m_{a}}\right|,
$$

где собственные значения $g_{i}^{l_{a}}$ являются простыми функциями энергии, зависящими только от разбиения спектра:

$$
\begin{array}{ll}
g_{i}^{l_{a}}(E)=\frac{1}{E-\varepsilon_{i}^{l_{a}}}, & i=1, \ldots, k_{l_{a}}, \quad \text { (15a) } \\
g_{i}^{l_{a}}(E)=\frac{1}{\delta_{i}^{l_{a}}}\left[\ln \left|\frac{E_{i-1}^{l_{a}}-E}{E_{i}^{l_{a}}-E}\right|-i \pi\left\{\theta\left(E_{i}^{l_{a}}-E\right)-\theta\left(E_{i-1}^{l_{a}}-E\right)\right\}\right], & i=k_{l_{a}}+1, \ldots, n_{l_{a}},
\end{array}
$$

где $\theta(\cdot)$ - тета-функция Хевисайда.

2.3. Пакетный базис для свободного гамильтониана $h_{0}^{a}$ • Гамильтониан $h_{0}^{a}$ в каждой парциальной волне $\left(L_{a}, \mu_{a}\right)$ имеет чисто непрерывный спектр $\left(\left(2 L_{a}+1\right)\right.$ кратно вырожденный по $\mu_{a}$ ), разбиение которого мы зададим как

$$
0=\mathcal{E}_{0}^{L_{a}}<\mathcal{E}_{1}^{L_{a}}<\cdots<\mathcal{E}_{j}^{L_{a}}<\cdots<\mathcal{E}_{N_{L_{a}}}^{L_{a}}, \quad \Delta_{j}^{L_{a}}=\mathcal{E}_{j}^{L_{a}}-\mathcal{E}_{j-1}^{L_{a}} .
$$

Построим из волновых функций свободного движения $\left|\psi_{0}\left(E, L_{a}\right)\right\rangle$ конечный набор свободных волновых пакетов $\left|X_{j}^{L_{a}}\right\rangle$ аналогично (10):

$$
\left|X_{j}^{L_{a} \mu_{a}}\right\rangle=\frac{1}{\sqrt{\Delta_{j}^{L_{a}}}} \int_{\mathcal{E}_{j-1}^{L_{a}}}^{\mathcal{E}_{j}^{L_{a}}} d E\left|\psi_{0}\left(E, L_{a} \mu_{a}\right)\right\rangle, \quad j=1, \ldots, N_{L_{a}} .
$$

Свойства свободных волновых пакетов аналогичны свойствам возмущенных волновых пакетов $\left|Z_{i}^{l_{a}}\right\rangle$ с тем лишь отличием, что у свободного гамильтониана дискретный спектр отсутствует. Состояния $\left|X_{j}^{L_{a}}\right\rangle$ образуют ортонормированный базис. Матрица гамильтониана $h_{0}^{a}$ диагональна в этом базисе, а ее собственные значения $\mathcal{E}_{i}^{* L_{a}}=\left(\mathcal{E}_{i-1}^{L_{a}}+\mathcal{E}_{i}^{L_{a}}\right) / 2$ являются средними точками интервалов разбиения.

Для проектора $\mathbb{J}^{L_{a} \mu_{a}}$ на подпространство свободных волновых пакетов для гамильтониана $h_{0}^{a}$, заданного формулой

$$
\mathbb{J}^{L_{a} \mu_{a}}=\sum_{j=1}^{N_{L_{a}}}\left|X_{j}^{L_{a} \mu_{a}}\right\rangle\left\langle X_{j}^{L_{a} \mu_{a}}\right|
$$

выполняется проекционное соотношение, аналогичное (12). 
Если использовать спектральное разложение двухчастичной свободной резольвенты $g_{0}^{(+)}(E)=\left[E+i 0-h_{0}^{a}\right]^{-1}$,

$$
g_{0}^{(+)}(E)=\sum_{L_{a}=0}^{\infty} \sum_{\mu=-L_{a}}^{L_{a}} \int_{0}^{\infty} d E_{2} \frac{\left|\psi_{0}\left(E_{2}, L_{a} \mu_{a}\right)\right\rangle\left\langle\psi_{0}\left(E_{2}, L_{a} \mu_{a}\right)\right|}{E+i 0-E_{2}}
$$

то легко получить ее проекцию на подпространство свободных волновых пакетов, которая имеет диагональный вид, аналогичный (14). Соответствующие собственные значения спроектированной свободной резольвенты определяются формулой (15б), в которой следует заменить параметры разбиения спектра гамильтониана $h_{a}$, см. формулу (9), на параметры разбиения спектра $h_{0}^{a}$, заданные в (16).

2.4. Собственное пакетное подпространство канала. Волновые функции состояний рассеяния трехчастичного гамильтониана канала $H_{a}$ являются произведениями функций $\left|\psi_{0}\left(E_{1}, L_{a} \mu_{a}\right)\right\rangle$ и $\left|\psi\left(E_{2}, l_{a} m_{a}\right)\right\rangle$. Из таких произведений составим волновые функции, отвечающие определенному значению полного орбитального момента $\Lambda$ и его проекции $M\left(\boldsymbol{\Lambda}=\mathbf{l}_{a}+\mathbf{L}_{a}\right)$ :

$$
\left\langle\boldsymbol{\xi}_{a}, \boldsymbol{\eta}_{a} \mid \psi\left(E_{1}, l_{a}\right), \psi_{0}\left(E_{2}, L_{a}\right), \Lambda M\right\rangle \equiv \psi\left(E_{1}, l_{a} ; \xi_{a}\right) \psi_{0}\left(E_{2}, L_{a} ; \eta_{a}\right) \mathcal{Y}_{l_{a} L_{a}}^{\Lambda M}\left(\hat{\boldsymbol{\xi}}_{a}, \hat{\boldsymbol{\eta}}_{a}\right),
$$

где шестимерные сферические гармоники $\mathcal{Y}_{l_{a} L_{a}}^{\Lambda M}$ являются сверткой шаровых функций $Y_{l_{a} m_{a}}\left(\hat{\boldsymbol{\xi}}_{a}\right)$ и $Y_{L_{a} \mu_{a}}\left(\hat{\boldsymbol{\eta}}_{a}\right)$, зависящих от координат Якоби.

Определим теперь стационарные ТВП как произведения соответствующих двухчастичных волновых пакетов, отвечающих субгамильтонианам $h_{a}$ и $h_{0}^{a}$ :

$$
\left\langle\boldsymbol{\xi}_{a}, \boldsymbol{\eta}_{a} \mid Z_{i}^{l_{a}}, X_{j}^{L_{a}}, \Lambda M\right\rangle=Z_{i}^{l_{a}}\left(\xi_{a}\right) X_{j}^{L_{a}}\left(\eta_{a}\right) \mathcal{Y}_{l_{a} L_{a}}^{\Lambda M}\left(\hat{\boldsymbol{\xi}}_{a}, \hat{\boldsymbol{\eta}}_{a}\right) .
$$

Для краткости положим $\left|Z_{i}^{l_{a}}, X_{j}^{L_{a}}, \Lambda M\right\rangle \equiv\left|S_{a}, \Lambda M\right\rangle$, где введен мультииндекс $S_{a}=$ $\left(i, l_{a}, j, L_{a}\right)$. Полное число $\mathcal{N}_{a}$ базисных функций определяется неравенствами

$$
0 \leqslant l_{a} \leqslant l_{a}^{\max }, \quad 0 \leqslant L_{a} \leqslant L_{a}^{\max }, \quad 1 \leqslant i \leqslant n_{l_{a}}, \quad 1 \leqslant j \leqslant N_{L_{a}} .
$$

Свойства ТВП в целом сходны со свойствами двухчастичных волновых пакетов. Легко показать, что они образуют ортонормированную систему,

$$
\left\langle S_{a}, \Lambda M \mid S_{a}^{\prime}, \Lambda M\right\rangle=\delta_{S_{a}, S_{a}^{\prime}},
$$

что следует из свойств ортонормированности двухчастичных волновых пакетов. Таким образом, проектор на подпространство волновых пакетов (с полным орбитальным моментом $\Lambda$ и его проекцией $M$ ) в канале $a$ следует определить как

$$
\mathbb{P}_{a}^{\Lambda M}=\sum_{S_{a}=1}^{\mathcal{N}_{a}}\left|S_{a}, \Lambda M\right\rangle\left\langle S_{a}, \Lambda M\right|
$$

Из определения ТВП следует, что трехчастичный проектор является суммой прямых произведений двухчастичных проекторов (11) и (18):

$$
\mathbb{P}_{a}^{\Lambda M}=\sum_{l_{a}, L_{a}} \sum_{m_{a}+\mu_{a}=M} C_{l_{a} m_{a}, L_{a} \mu_{a}}^{\Lambda M} \mathbb{I}^{l_{a} m_{a}} \otimes \mathbb{J}^{L_{a} \mu_{a}},
$$


где $C$ - коэффициенты Клебша-Гордана. В дальнейшем для упрощения записи будем опускать индекс $M$.

Аналогично двухчастичному случаю будут выполняться проекционные правила для собственных функций гамильтониана $H_{a}$ :

$$
\begin{aligned}
& \mathbb{P}_{a}^{\Lambda}\left|\hat{\psi}_{k}^{l_{a}}, \psi_{0}\left(E_{2}, L_{a}\right), \Lambda\right\rangle= \begin{cases}\frac{\left|Z_{k}^{l_{a}}, X_{j}^{L_{a}}, \Lambda\right\rangle}{\sqrt{\Delta_{j}^{L_{a}}},} & E_{2} \in\left(\mathcal{E}_{j-1}^{L_{a}}, \mathcal{E}_{j}^{L_{a}}\right), \\
0, & E_{2} \notin\left(0, \mathcal{E}_{N_{L_{a}}}^{L_{a}}\right) ;\end{cases} \\
& \mathbb{P}_{a}^{\Lambda}\left|\psi\left(E_{1}, l_{a}\right), \psi_{0}\left(E_{2}, L_{a}\right), \Lambda\right\rangle= \begin{cases}\frac{\left|Z_{i}^{l_{a}}, X_{j}^{L_{a}}, \Lambda\right\rangle}{\sqrt{\delta_{i}^{l_{a}} \Delta_{j}^{L_{a}}},} & \left(\begin{array}{l}
E_{1} \in\left(E_{i-1}^{l_{a}}, E_{i}^{l_{a}}\right), \\
E_{2} \in\left(\mathcal{E}_{j-1}^{L_{a}}, \mathcal{E}_{j}^{L_{a}}\right)
\end{array}\right), \\
0, & \left(\begin{array}{l}
E_{1} \notin\left(0, E_{n_{l_{a}}}^{l_{a}}\right) \\
E_{2} \notin\left(0, \mathcal{E}_{N_{L_{a}}}^{L_{a}}\right)
\end{array}\right) .\end{cases}
\end{aligned}
$$

Следующее важное свойство ТВП состоит в том, что они являются собственными векторами проекции канального гамильтониана на пакетное подпространство:

$$
\mathbb{P}_{a}^{\Lambda} H_{a} \mathbb{P}_{a}^{\Lambda}\left|Z_{i}^{l_{a}}, X_{j}^{L_{a}}, \Lambda\right\rangle=\left(E_{i}^{* l_{a}}+\mathcal{E}_{j}^{* L_{a}}\right)\left|Z_{i}^{l_{a}}, X_{j}^{L_{a}}, \Lambda\right\rangle .
$$

Это следует из диагональности проекций субгамильтонианов $h_{a}$ и $h_{0}^{a}$ на соответствующие подпространства двухчастичных волновых пакетов. При этом собственные значения пакетной проекции канального гамильтониана являются суммами собственных значений соответствующих пакетных проекций гамильтонианов $h_{a}$ и $h_{0}^{a}$. Будем называть далее рассматриваемое подпространство ТВП собственным nакетным подпространством канала а.

Исходя из перечисленных спектральных свойств ТВП, несложно получить удобные конечномерные представления для проекций канальных резольвент на их собственные пакетные подпространства.

2.5. Пакетная проекция канальной резольвенты. Определим резольвенту гамильтониана канала $H_{a}$,

$$
G^{a}(\zeta)=\left[\zeta-H_{a}\right]^{-1}, \quad a=1,2,3,
$$

которая согласно [18] является сверткой двухчастичных резольвент:

$$
G^{a}(E)=\frac{1}{2 \pi i} \int_{-\infty}^{+\infty} d \varepsilon g_{a}^{(+)}(E-\varepsilon) g_{0}^{(+)}(\varepsilon) .
$$

Подставим в эту формулу спектральные разложения (13) и (19) двухчастичных резольвент $g_{a}^{(+)}$и $g_{0}^{(+)}$, выполним интегрирование и перейдем в подынтегральном выражении к трехчастичным собственным функциям (20) гамильтониана $H_{a}$ с определенным полным угловым моментом. В результате получим выражение для резольвенты канала $a$ в виде суммы двух членов:

$$
G^{a}(E)=R^{a}(E)+Q^{a}(E),
$$

5 Теоретическая и математическая физика, т. 150, № 3, 2007 г. 
где

$$
\begin{gathered}
R^{a}=\sum_{\Lambda, M} \sum_{l_{a}, L_{a}} \sum_{i=1}^{k_{l_{a}}} \int_{0}^{\infty} d E_{2} \frac{\left|Z_{i}^{l_{a}}, \psi_{0}\left(E_{2}, L_{a}\right), \Lambda M\right\rangle\left\langle Z_{i}^{l_{a}}, \psi_{0}\left(E_{2}, L_{a}\right), \Lambda M\right|}{E+i 0-\varepsilon_{i}^{l_{a}}-E_{2}}, \\
Q^{a}=\sum_{\Lambda, M} \sum_{l_{a}, L_{a}} \int_{0}^{\infty} d E_{1} \int_{0}^{\infty} d E_{2} \frac{\left|\psi\left(E_{1}, l_{a}\right), \psi_{0}\left(E_{2}, L_{a}\right), \Lambda M\right\rangle\left\langle\psi\left(E_{1}, l_{a}\right), \psi_{0}\left(E_{2}, L_{a}\right), \Lambda M\right|}{E+i 0-E_{1}-E_{2}}
\end{gathered}
$$

- так называемые bound-continuum (BC) и continuum-continuum (CC) части резольвенты, соответственно. Применим к полученным формулам проектирование на собственное пакетное подпространство канала $a$. Тогда для ВС-части резольвенты получим следующее конечномерное диагональное представление:

$$
\mathbb{P}_{a}^{\Lambda} R^{a} \mathbb{P}_{a}^{\Lambda} \equiv \mathbb{R}^{a}(E)=\sum_{S_{a}}\left|S_{a}, \Lambda\right\rangle\left[R^{a}(E)\right]_{S_{a}}\left\langle S_{a}, \Lambda\right|,
$$

где собственные значения $\left[R^{a}(E)\right]_{S_{a}}$ определяются формулой

$$
\left[R^{a}(E)\right]_{S_{a}}=\frac{1}{\Delta_{j}^{L_{a}}} \int_{\mathcal{E}_{j-1}^{L_{a}}}^{\mathcal{E}_{j}^{L_{a}}} \frac{d E_{2}}{E+i 0-\varepsilon_{k}^{l_{a}}-E_{2}}, \quad S_{a}=\left(k, l_{a}, j, L_{a}\right)
$$

Вычисляя интеграл, получаем явные выражения для реальной и мнимой частей собственных значений ВС-части проекции канальной резольвенты:

$$
\begin{aligned}
\operatorname{Re}\left[R^{a}(E)\right]_{S_{a}} & =\frac{1}{\Delta_{j}^{L_{a}}} \ln \left|\frac{\mathcal{E}_{j-1}^{L_{a}}+\varepsilon_{k}^{l_{a}}-E}{\mathcal{E}_{j}^{L_{a}}+\varepsilon_{k}^{l_{a}}-E}\right|, \\
\operatorname{Im}\left[R^{a}(E)\right]_{S_{a}} & =-\frac{\pi}{\Delta_{j}^{L_{a}}}\left\{\theta\left(\mathcal{E}_{j}^{L_{a}}+\varepsilon_{k}^{l_{a}}-E\right)-\theta\left(\mathcal{E}_{j-1}^{L_{a}}+\varepsilon_{k}^{l_{a}}-E\right)\right\} .
\end{aligned}
$$

Легко видеть, что формулы для собственных значений ВС-части канальной резольвенты аналогичны формулам (15a), (15б) для собственных значений двухчастичной резольвенты с тем отличием, что граничные точки $\mathcal{E}_{j}^{L_{a}}$ разбиения спектра свободного гамильтониана $h_{0}^{a}$ сдвигаются на энергии связи $\varepsilon_{k}^{l a}$ гамильтониана $h_{a}$ для парного углового момента $l_{a}$.

Выражение для проекции СС-части резольвенты также имеет диагональный вид и, что особенно важно, задается явной формулой

$$
\mathbb{Q}^{a}(E)=\sum_{S_{a}^{\prime}}\left|S_{a}^{\prime}, \Lambda\right\rangle\left[Q^{a}(E)\right]_{S_{a}^{\prime}}\left\langle S_{a}^{\prime}, \Lambda\right|,
$$

где собственные значения определяются двукратным интегралом по энергетическим интервалам разбиений двухчастичных спектров (9) и (16):

$$
\left[Q^{a}(E)\right]_{S_{a}^{\prime}}=\frac{1}{\delta_{i}^{l_{a}} \Delta_{j}^{L_{a}}} \int_{E_{i-1}^{l_{a}}}^{E_{i}^{l_{a}}} \int_{\mathcal{E}_{j-1}^{L_{a}}}^{\mathcal{E}_{j}^{L_{a}}} \frac{d E_{1} d E_{2}}{E+i 0-E_{1}-E_{2}} .
$$

В последних двух формулах мультииндекс $S_{a}$ помечен штрихом, для того чтобы подчеркнуть, что в СС-части резольвенты суммирование ведется по всем пакетным состояниям, не вошедшим в ВС-часть. 
Выполняя интегрирование в формуле (33), получаем следующие явные выражения для реальных частей собственных значений СС-части проекции канальной резольвенты:

$$
\begin{aligned}
\operatorname{Re}\left[Q^{a}(E)\right]_{S_{a}^{\prime}}= & \frac{1}{\delta_{i}^{l_{a}} \Delta_{j}^{L_{a}}}\left\{\left(\Delta+\Delta_{-}\right) \ln \left|\Delta+\Delta_{-}\right|+\left(\Delta-\Delta_{-}\right) \ln \left|\Delta-\Delta_{-}\right|\right\}- \\
& -\frac{1}{\delta_{i}^{l_{a}} \Delta_{j}^{L_{a}}}\left\{\left(\Delta+\Delta_{+}\right) \ln \left|\Delta+\Delta_{+}\right|+\left(\Delta-\Delta_{+}\right) \ln \left|\Delta-\Delta_{+}\right|\right\},
\end{aligned}
$$

где использованы обозначения

$$
\Delta \equiv E_{i}^{* l_{a}}+\mathcal{E}_{j}^{* L_{a}}-E, \quad \Delta_{-} \equiv \frac{\delta_{i}^{l_{a}}-\Delta_{j}^{L_{a}}}{2}, \quad \Delta_{+} \equiv \frac{\delta_{i}^{l_{a}}+\Delta_{j}^{L_{a}}}{2}, \quad S_{a}^{\prime}=\left(i, l_{a}, j, L_{a}\right) .
$$

При этом мнимые части собственных значений также выражаются в явном виде:

$$
\begin{aligned}
\operatorname{Im}\left[Q^{a}(E)\right]_{S_{a}^{\prime}}=- & \frac{\pi}{\delta_{i}^{l_{a}} \Delta_{j}^{L_{a}}}\left\{\left(\Delta+\Delta_{+}\right) \theta\left(\Delta+\Delta_{+}\right)+\left(\Delta-\Delta_{+}\right) \theta\left(\Delta-\Delta_{+}\right)-\right. \\
& \left.-\left(\Delta+\Delta_{-}\right) \theta\left(\Delta+\Delta_{-}\right)-\left(\Delta-\Delta_{-}\right) \theta\left(\Delta-\Delta_{-}\right)\right\} .
\end{aligned}
$$

Итак, используя свойства трехчастичных волновых пакетов и их прямую связь с точными функциями непрерывного спектра, мы получили, как и в двухчастичном случае, диагональную матрицу для проекции канальной резольвенты на собственное пакетное подпространство. Собственные значения этой матрицы являются сравнительно простыми функциями энергии, зависящими только от энергий связанных состояний подсистем и разбиений непрерывных спектров. Важно подчеркнуть, что при таком подходе пакетная проекция точной трехчастичной канальной резольвенты задается по существу сверткой (по обеим координатам Якоби) двух одночастичных матричных резольвент, энергетические особенности которых выделяются в явном виде и интегрируются. Тем самым энергетическая зависимость как действительных, так и мнимых частей этих собственных значений явно выделяется в аналитическом виде.

Исследуем теперь, как можно использовать описанную пакетную технику для решения трехчастичной задачи.

\section{3. РАССЕЯНИЕ СОСТАВНОЙ ЧАСТИЦЫ В ПОЛЕ ЯДРА}

3.1. Амплитуда упругого рассеяния. Рассмотрим задачу рассеяния связанной пары $\{23\}$ на ядре (частице 1). Если каждая из частиц-снарядов 2 и 3 не образует связанных состояний или ярко выраженных резонансов с ядром-мишенью, а также если мнимая часть потенциалов взаимодействия $V_{2}$ и $V_{3}$ не является малой, то либо каналы перестройки вовсе отсутствуют, либо их влияние на амплитуду упругого рассеяния снаряда слабое. В этом случае для нахождения амплитуды рассеяния можно воспользоваться простым приближением и учитывать граничные условия только в одном асимптотическом канале ${ }^{1)}$. При этом развал падающей

\footnotetext{
1) В ядерной физике эта ситуация встречается очень часто при рассмотрении упругого и неупругого рассеяния дейтронов, тритонов и других легких ядер в поле тяжелого ядра-мишени.
} 
частицы трактуется как возбуждение ее внутреннего состояния в континуум. Будем считать для простоты, что в подсистеме $\{23\}$ имеется только одно $s$-волновое связанное состояние $\left|Z_{0}\right\rangle$ с энергией $\varepsilon_{0}$.

Поскольку каналы перестройки мы не учитываем явно, для нахождения полной резольвенты $G(E)$ можно использовать одно уравнение Липпмана-Швингера, связывающее $G(E)$ и канальную резольвенту $G^{1}(E)$ :

$$
G(E)=G^{1}(E)+G^{1}(E)\left(V_{2}+V_{3}\right) G(E) .
$$

Как уже указывалось выше, для вычисления амплитуды упругого рассеяния (6) достаточно знать ядро полной резольвенты системы лишь в некоторой ограниченной области конфигурационного пространства. Применим к уравнению (36) справа и слева оператор проектирования $\mathbb{P}_{1}$ на собственное пакетное подпространство канала. Заменим короткодействующие потенциалы взаимодействия частиц с ядроммишенью эффективным оператором взаимодействия в пакетном подпространстве

$$
\widehat{V}=\mathbb{P}_{1}\left(V_{2}+V_{3}\right) \mathbb{P}_{1}
$$

В результате мы получим уравнение с конечномерным ядром для проекции полной резольвенты $\mathbb{G}(E) \equiv \mathbb{P}_{1} G(E) \mathbb{P}_{1}$ :

$$
\mathbb{G}(E)=\mathbb{G}^{1}+\mathbb{G}^{1} \widehat{V} \mathbb{G}(E) .
$$

Решение этого уравнения имеет вид

$$
\mathbb{G}(E)=\left(\mathbb{G}^{1}(E)^{-1}-\widehat{V}\right)^{-1} .
$$

Заменяя в формуле (7) для амплитуды упругого рассеяния оператор внешнего взаимодействия $U_{1}=V_{2}+V_{3}$ оператором (37) и учитывая проекционные свойства пакетного проектора, получаем для этой амплитуды компактное выражение

$$
A_{\mathrm{el}}(E, \Lambda)=\frac{\left\langle S_{0}, \Lambda|\widehat{V}+\widehat{V} \mathbb{G}(E) \widehat{V}| S_{0}, \Lambda\right\rangle}{\Delta_{j}^{\Lambda}} .
$$

Здесь $\left|S_{0}, \Lambda\right\rangle$ - это трехчастичный волновой пакет, который является произведением функции связанного состояния $\left|Z_{0}\right\rangle$ подсистемы $\{23\}$ и свободного пакета $\left|X_{j}^{\Lambda}\right\rangle$ как функции от координаты движения центра масс пары относительно ядра-мишени. При этом полная энергия системы $E$ принадлежит интервалу $\left(\mathcal{E}_{j-1}^{\Lambda}+\varepsilon_{0}, \mathcal{E}_{j}^{\Lambda}+\varepsilon_{0}\right)$ с шириной $\Delta_{j}^{\Lambda}$.

Окончательно амплитуда упругого рассеяния определяется как диагональный элемент:

$$
A_{\mathrm{el}}(E, \Lambda)=\frac{\left[\mathbf{V}+\mathbf{V}\left(\left(\mathbf{G}^{1}\right)^{-1}-\mathbf{V}\right)^{-1} \mathbf{V}\right]_{S_{0}, S_{0}}}{\Delta_{j}^{\Lambda}}=\frac{\left[\mathbf{V}^{-1}-\mathbf{G}^{1}\right]_{S_{0}, S_{0}}^{-1}}{\Delta_{j}^{\Lambda}},
$$

где жирным шрифтом обозначены матрицы соответствующих операторов в пакетном базисе. Итак, в рассмотренном простом случае упругого рассеяния составной частицы на ядре (когда используется пакетный базис лишь в одном наборе координат Якоби) схема решения состоит из следующих шагов: 
1) определение пакетных спектров двухчастичных гамильтонианов $h_{1}$ и $h_{0}^{1}$ и построение на их основе собственного пакетного подпространства канального гамильтониана $H_{1}$;

2) нахождение элементов матрицы канальной резольвенты по формулам (32)-(35) и матрицы эффективного потенциала взаимодействия (37);

3) нахождение амплитуды по формуле (41).

Промежуточные вычисления в этой схеме можно существенно упростить, если использовать соответствие между стационарными волновыми пакетами и функциями псевдосостояний гамильтониана, установленное нами в работах [16], [17]. Под псевдосостояниями мы понимаем здесь собственные состояния матрицы гамильтониана в некотором $L_{2}$-базисе, отвечающие области непрерывного спектра этого гамильтониана. В [16] было показано, что функции псевдосостояний являются аппроксимациями стационарных волновых пакетов, а не точных собственных волновых функций рассеяния рассматриваемого гамильтониана. Поэтому можно получать разложения функций пакетного базиса по некоторому $L_{2}$-базису путем всего одной диагонализации матрицы гамильтониана в этом базисе.

Следует подчеркнуть, что формула (41) для амплитуды трехчастичного упругого рассеяния аналогична формуле для элементов $T$-матрицы, использовавшейся для решения двухчастичной задачи [16]. Таким образом, общее усложнение схемы решения рассмотренной трехчастичной задачи по сравнению с двухчастичной связано только с необходимостью построения дискретизованных спектров двух гамильтонианов вместо одного (и соответственно с увеличением размерностей используемых матриц). В остальном алгоритм решения остается прежним. Простота решения связана с диагональностью представления в пакетном базисе трехчастичной канальной резольвенты, а также с простотой представления трехчастичного взаимодействия в таком базисе.

Отметим, что по сути описанный здесь метод пакетной дискретизации континуума (МПДК) близок к различным вариантам метода сильной связи каналов, широко используемого для решения аналогичных задач теории рассеяния. Наиболее известен из них в настоящее время CDCC-метод [9]-[11]. Он основан на разложении полной волновой функции системы по компонентам, отвечающим различным состояниям дискретизованного спектра внутреннего гамильтониана пары $\{23\}$. Для таких компонент из трехчастичного уравнения Шредингера получается CDCCсистема дифференциальных уравнений второго порядка, связывающая функцию упругого рассеяния с функциями каналов развала. Таким образом, дискретизация континуума в CDCC-методе выполняется лишь частично: вводится пакетное представление только по одной радиальной переменной $\xi$, тогда как по другой переменной $\eta$ необходимо решать громоздкую систему дифференциальных уравнений с условием сшивки, учитываюшим закон сохранения энергии $\mathcal{E}_{j}=E-E_{i}$.

Основное отличие нашего подхода от CDCC-метода состоит именно в использовании полной дискретизации трехчастичного спектра (т.е. мы добавляем дискретизацию спектра гамильтониана свободного движения $\left.h_{0}^{1}\right)$, при этом волновая функция системы фактически представляется алгебраическими коэффициентами разложения по пакетному базису, определяющему проектор $\mathbb{P}_{1}$. В итоге вместо громоздкой системы связанных дифференциальных уравнений с граничными условиями на бесконечности решается всего одно интегральное уравнение, которое в итоге сводится 
к матричному, причем элементы матриц задаются явными аналитическими формулами, что позволяет кардинально упростить и ускорить весь процесс решения.

Важно, что на основе МПДК можно найти также и амплитуду развала частицы $\{23\}$. Эта амплитуда определяется выражением

$A\left(E_{1}, E_{2}, E\right)=\left\langle\psi^{(-)}\left(E_{1}\right), \psi_{0}\left(E_{2}\right), \Lambda\left|\left(V_{2}+V_{3}\right)+\left(V_{2}+V_{3}\right) G\left(V_{2}+V_{3}\right)\right| Z_{0}, \psi_{0}\left(E-\varepsilon_{0}, \Lambda\right)\right\rangle$,

где $\psi^{(-)}-$функция рассеяния для двухчастичного гамильтониана $h_{1}$, отвечающая граничному условию в виде сходящихся волн. В (42) выполняется условие сохранения энергии $E_{1}+E_{2}=E$. Видно, что здесь уже нет полного "обрезания" резольвенты по всем пространственным переменным, поскольку в левой “обкладке" стоят неубывающие функции рассеяния. Однако, если проинтегрировать выражение (42) по узким полосам разбиений энергий $E_{1}$ и $E_{2}$, мы получим некоторое усредненное значение амплитуды развала. При этом слева вместо точных функций рассеяния появятся соответствующие волновые пакеты, и ядро резольвенты в (42) снова будет полностью “обрезано” за счет убывания этих пакетных функций.

Таким образом, для вычисления искомой амплитуды развала можно воспользоваться снова простой матричной формулой

$$
A\left(E_{1}, E_{2}, E\right) \approx \frac{e^{i \phi}\left[\mathbf{V}+\mathbf{V}\left(\left(\mathbf{G}^{1}\right)^{-1}-\mathbf{V}\right)^{-1} \mathbf{V}\right]_{S, S_{0}}}{\sqrt{\delta_{i^{\prime}} \Delta_{j^{\prime}} \Delta_{j}}},
$$

где состояние $\left|S_{0}\right\rangle$ отвечает функции начального состояния, а конечное состояние задается пакетом $|S, \Lambda\rangle \equiv\left|Z_{i^{\prime}}^{l^{\prime}}, X_{j^{\prime}}^{L^{\prime}}, \Lambda\right\rangle$, для которого $E_{1} \in\left(E_{i^{\prime}-1}^{l^{\prime}}, E_{i^{\prime}}^{l^{\prime}}\right)$ и $E_{2} \in$ $\left(E_{j^{\prime}-1}^{L^{\prime}}, E_{j^{\prime}}^{L^{\prime}}\right) ; \phi-$ парциальный фазовый сдвиг, отвечающий волновой функции $\psi^{(+)}\left(E_{1}\right)$. Легко видеть, что амплитуды упругого рассеяния и развала определяются из одной и той же матрицы $\mathbf{T}=\mathbf{V}+\mathbf{V}\left(\mathbf{G}_{1}^{-1}-\mathbf{V}\right)^{-1} \mathbf{V}$, причем амплитуда упругого рассеяния находится из диагональных элементов, а амплитуда развала из недиагональных элементов матрицы трехчастичного оператора рассеяния.

3.2. Численная иллюстрация. В качестве примера численной реализации МПДК рассмотрим задачу рассеяния дейтрона на тяжелом ядре, которая многократно использовалась в качестве теста для развитых ранее методов (см., например, работы [9], [10], [19] и ссылки в них на более ранние исследования). Взаимодействие нуклонов между собой задается простым гауссовым потенциалом

$$
V_{1}\left(r_{23}\right)=-V_{0} \exp \left(-\gamma r_{23}^{2}\right)
$$

при значениях параметров $V_{0}=66.99 \mathrm{MэВ} \mathrm{и} \gamma=0.415 \Phi_{\mathrm{M}}{ }^{-2}$. В этом случае в подсистеме $\{23\}$ есть одно $s$-волновое связанное состояние - "дейтрон" с энергией $\varepsilon_{0}=-2.226$ МэВ. Взаимодействия нейтрона и протона с ядром-мишенью описываются одинаковыми комплексными оптическими потенциалами

$$
V_{2}\left(r_{13}\right)=-U_{0} \exp \left(-\delta r_{13}^{2}\right), \quad V_{3}\left(r_{12}\right)=-U_{0} \exp \left(-\delta r_{12}^{2}\right)
$$

с параметрами $U_{0}=(50+5 i) \mathrm{M \ni B}, \delta=0.0625 \Phi_{\mathrm{M}^{-2}}$. В расчетах используется значение кинематического параметра $\hbar^{2} / m=41.47 \mathrm{MэB} \cdot \Phi_{\mathrm{M}}{ }^{-2}$, где $m$ - масса нуклона. В данной модели учитывается только $s$-волновое парное взаимодействие между нуклонами, т.е. $l=0, L=\Lambda$. 
Введем координаты Якоби в канале 1:

$$
\boldsymbol{\xi}=\mathbf{r}_{2}-\mathbf{r}_{3}, \quad \boldsymbol{\eta}=\mathbf{r}_{1}-\frac{1}{2}\left(\mathbf{r}_{2}+\mathbf{r}_{3}\right)
$$

где $\mathbf{r}_{i}$ - радиус-векторы частиц. Расстояния между частицами задаются через координаты Якоби следующим образом:

$$
r_{23}=\xi, \quad r_{13}=\left|\frac{1}{2} \boldsymbol{\xi}+\boldsymbol{\eta}\right|, \quad r_{12}=\left|\frac{1}{2} \boldsymbol{\xi}-\boldsymbol{\eta}\right| .
$$

Как уже упоминалось выше, в работах [16], [17] нами было показано, что в качестве функций пакетного базиса можно использовать волновые функции псевдосостояний, полученных путем диагонализации матрицы парного гамильтониана в подходящем $L_{2}$-базисе. Воспользуемся этим для построения пакетного базиса (10) двухчастичного нуклон-нуклонного гамильтониана $h_{1}$. Будем считать, что

$$
\left|Z_{i}^{l}\right\rangle \approx\left|\widetilde{Z}_{i}^{l}\right\rangle=\sum_{k=1}^{K} D_{k}^{i}\left|\phi_{k l}\right\rangle
$$

где $\left|\widetilde{Z}_{i}^{l}\right\rangle$ - функции псевдосостояний, полученные в результате диагонализации матрицы гамильтониана $h_{1}$ в некотором базисе $\left\{\left|\phi_{k l}\right\rangle\right\}_{k=1}^{K}$. В набор состояний (48) входит и связанное состояние подсистемы $\{23\}$, аппроксимация которого получается на основании единой процедуры диагонализации.

В качестве $L_{2}$-базиса для построения спектров дискретизации двухчастичного гамильтониана $h_{1}$ удобно использовать базис гауссова типа,

$$
\phi_{k}(\xi)=e^{-\alpha_{k} \xi^{2}}, \quad k=1, \ldots, K,
$$

с масштабными параметрами $\alpha_{k}$, определяемыми на основе обобщенного распределения Чебышева,

$$
\alpha_{k}=\alpha_{0}\left[\operatorname{tg}\left(\frac{2 k-1}{4 K} \pi\right)\right]^{t}, \quad k=1, \ldots, K,
$$

где $\alpha_{0}$ - общий масштабный параметр базиса, и значение параметра $t$ определяет степень "разреженности" нелинейных параметров базиса $\alpha_{k}$.

Для построения пакетного базиса гамильтониана $h_{0}^{1}$, описывающего свободное движение связанной пары $\{23\}$ относительно ядра-мишени, будем использовать эквидистантное разбиение спектра $\left[0, E_{m}\right]$ на $N$ частей (для каждого углового момента $L)$. В качестве пакетных функций будем использовать их приближенное выражение через точные функции свободного движения на соответствующем $j$-м энергетическом интервале:

$$
X_{j}^{L}(\eta) \approx \sqrt{\frac{\Delta m}{\hbar^{2}}} \frac{J_{L+1 / 2}\left(K_{j} \eta\right)}{\sqrt{\eta}}, \quad K_{j}=\sqrt{\frac{2 m \mathcal{E}_{j}^{*}}{\hbar^{2}}},
$$

где $J_{L+1 / 2}(x)$ - цилиндрическая функция Бесселя, параметр $\Delta=E_{\max } / N$ задает ширину энергетических полос разбиения спектра. 
ТВП, составляющие проектор $\mathbb{P}_{1}^{L}$, являются произведениями двухчастичных волновых пакетов. Представление двухчастичных волновых пакетов, заданное с помощью формул (48) и (51), приводит к тому, что матричные элементы потенциала взаимодействия (37) в собственном пакетном базисе полностью вычисляются аналитически.

Матричные элементы канальной резольвенты $G^{1}$ вычисляются по формулам (32)(35), в которых в качестве параметров дискретизации двухчастичного спектра нуклон-нуклонного гамильтониана $h_{1}$ используются параметры спектра псевдосостояний, найденные в результате диагонализации матрицы этого гамильтониана в гауссовом базисе.

После того как элементы матриц $\mathbf{V}$ и $\mathbf{G}^{1}$ вычислены, парциальные фазовые сдвиги $\delta_{L}(E)$ упругого рассеяния дейтрона на ядре находятся из формулы (41) с учетом связи амплитуды упругого рассеяния с $S$-матрицей:

$$
A(E, L)=\frac{1-S_{L}(E)}{2 \pi i}, \quad S_{L}(E)=\eta_{L} e^{2 i \delta_{L}(E)}, \quad \eta_{L}=\left|S_{L}(E)\right| .
$$

Заметим, что для дальнейшего упрощения расчетов можно несколько усовершенствовать схему, описанную выше. С помощью известного проекционного формализма Фешбаха можно свести рассматриваемую задачу упругого рассеяния составной частицы к двухчастичной задаче с эффективным взаимодействием, включающим в себя все внутренние возбуждения падающей частицы и зависящим от энергии [20]. При вычислении такого эффективного взаимодействия используется спроектированная на состояния континуума трехчастичная резольвента системы, которую мы вычислили раньше в пакетном представлении. Полная трехчастичная задача в таком подходе разбивается на два этапа:

- нахождение оператора эффективного взаимодействия;

- решение двухчастичной задачи рассеяния с найденным эффективным взаимодействием.

В результате такой модификации достигается значительное уменьшение размерности используемого трехчастичного базиса. В частности, на первом этапе используется базис свободных волновых пакетов $\left\{\chi_{i}\right\}$ сравнительно небольшой размерности $N_{\text {int }}$, так что полная размерность трехчастичного базиса равна $K \cdot N_{\text {int }}$. На втором этапе при решении задачи рассеяния с эффективным нелокальным потенциалом используется другой двухчастичный базис $\left\{\chi_{j}\right\}$ большей размерности $N_{\text {ext }}$.

В данной работе мы не будем подробно описывать детали этой схемы и исследовать сходимость результатов, полученных в пакетном подходе, к точным значениям в зависимости от размерности и типа используемого базиса. Этим вопросам будет посвящена отдельная работа. Мы приведем здесь только полученные результаты и сравним их с аналогичными результатами прямой матричной задачи.

В табл. 1 даны значения парциальных фазовых сдвигов $\delta_{L}$ и параметров неупругости $\eta_{L}$ для энергии падающего дейтрона $E_{d}=22.9 \mathrm{MэB}$, полученные на основе МПДК для различных значений полного момента $L=0 \div 15$ при использовании пакетного базиса разной размерности. Для пакетной аппроксимации нуклоннуклонного спектра использовались следующие параметры чебышевской сетки для 
построения гауссова базиса: $K=20, t=3, \alpha_{0}=0.1 \Phi_{\mathrm{M}^{-2}}$. При дискретизации спектра гамильтониана свободного движения центра масс дейтрона использовалось значение максимальной энергии $E_{\max }=300 \mathrm{MэB}$, при этом весь непрерывный спектр свободного движения делился на $N_{\text {int }}$ бинов $\left(N_{\text {int }}=30,50,70,100\right.$ - размерность внутреннего базиса). Размерность внешнего базиса считалась фиксированной: $N_{\text {ext }}=300$. Как следует из таблицы, базис размерности $N_{\text {int }}=30$ уже вполне достаточен для хорошей аппроксимации парциальных фазовых сдвигов $(d-A)$-рассеяния.

ТАБлицА 1. Парциальные фазовые сдвиги упругого рассеяния $\delta_{L}$ и коэффициенты неупругости $\eta_{L}$, полученные на основе МПДК, при энергии дейтрона $E_{d}=22.9$ МэВ для различных парциальных волн $L$ при разных размерностях $N_{\text {int }}$ базиса свободных волновых пакетов.

\begin{tabular}{|c|rc|rc|rc|rc|}
\hline \multirow{2}{*}{$\mathrm{L}$} & \multicolumn{2}{|c|}{$N_{\text {int }}=30$} & \multicolumn{2}{|c|}{$N_{\text {int }}=50$} & \multicolumn{2}{|c|}{$N_{\text {int }}=70$} & \multicolumn{2}{|c|}{$N_{\text {int }}=100$} \\
\cline { 2 - 10 } & $\delta_{L}$ & $\eta_{L}$ & $\delta_{L}$ & $\eta_{L}$ & $\delta_{L}$ & $\eta_{L}$ & $\delta_{L}$ & $\eta_{L}$ \\
\hline 0 & 70 & 0.22 & 70 & 0.22 & 67 & 0.21 & 67 & 0.22 \\
\hline 1 & 64 & 0.22 & 65 & 0.23 & 62 & 0.20 & 63 & 0.21 \\
\hline 2 & 51 & 0.21 & 50 & 0.24 & 52 & 0.20 & 52 & 0.21 \\
\hline 3 & 32 & 0.21 & 32 & 0.22 & 34 & 0.21 & 35 & 0.21 \\
\hline 4 & 10 & 0.19 & 11 & 0.21 & 8 & 0.22 & 9 & 0.21 \\
\hline 5 & -20 & 0.22 & -20 & 0.24 & -22 & 0.21 & -22 & 0.22 \\
\hline 6 & -63 & 0.23 & -62 & 0.23 & -63 & 0.22 & -62 & 0.22 \\
\hline 7 & 69 & 0.26 & 71 & 0.23 & 67 & 0.24 & 69 & 0.23 \\
\hline 8 & 14 & 0.34 & 13 & 0.30 & 12 & 0.32 & 12 & 0.31 \\
\hline 9 & -45 & 0.41 & -47 & 0.41 & -46 & 0.42 & -46 & 0.42 \\
\hline 10 & 71 & 0.56 & 72 & 0.57 & 72 & 0.55 & 72 & 0.56 \\
\hline 11 & 34 & 0.77 & 35 & 0.77 & 34 & 0.76 & 35 & 0.77 \\
\hline 12 & 17 & 0.89 & 17 & 0.89 & 17 & 0.88 & 17 & 0.89 \\
\hline 13 & 8.5 & 0.95 & 9 & 0.95 & 9 & 0.94 & 9 & 0.94 \\
\hline 14 & 4 & 0.97 & 4.5 & 0.98 & 4.4 & 0.97 & 4.5 & 0.97 \\
\hline 15 & 2 & 0.99 & 2 & 0.99 & 2 & 0.99 & 2 & 0.99 \\
\hline
\end{tabular}

Для сравнения в табл. 2 приведены фазы и коэффициенты неупругости, полученные ранее с помощью других методов. Среди них мы взяли CDCC-метод, вариационный метод (BM), а также метод конечных элементов (МКЭ). Как уже упоминалось выше, в CDCC-методе [9] используется дискретизация непрерывного спектра двухчастичного нуклон-нуклонного гамильтониана путем разбиения этого спектра на полосы, в каждой из которых строится приближенная функция возбужденного нуклон-нуклонного состояния ("возбужденного" дейтрона). Далее полная трехчастичная волновая функция рассеяния раскладывается по базисным функциям такого возбужденного дейтрона и в итоге получается система связанных дифференциальных уравнений для компонент волновой функции в разных каналах. BM [10] (который в настоящее время называется методом псевдосостояний) отличается от CDCC-метода лишь тем, что при дискретизации нуклон-нуклонного непрерывного спектра используется базис псевдосостояний гамильтониана $h_{1}$ в некотором $L_{2}$-базисе. Далее схема решения полностью аналогична схеме CDCC-метода. 
В МКЭ [19] используется трехчастичное уравнение Шредингера для рассматриваемой системы в гиперсферических координатах, а получившаяся система дифференциальных уравнений решается методом конечных элементов. В работе [19] МКЭ использовался в качестве независимой проверки для CDCC-метода и ВМ. Значения фазовых сдвигов и коэффициентов неупругости для CDCC-метода, ВM и МKЭ взяты из работы [19].

ТАБлицА 2. Парциальные фазовые сдвиги упругого рассеяния $\delta_{L}$ и коэффициенты неупругости $\eta_{L}$, полученные на основе МПДК и других методов, при энергии дейтрона $E_{d}=22.9 \mathrm{MэВ} \mathrm{для} \mathrm{разных} \mathrm{парциальных} \mathrm{волн} L$.

\begin{tabular}{|c|rc|rc|rc|rc|}
\hline \multirow{2}{*}{$\mathrm{L}$} & \multicolumn{2}{|c|}{ МПДК } & \multicolumn{2}{|c|}{ СDСС } & \multicolumn{2}{|c|}{ ВM } & \multicolumn{2}{|c|}{ MKЭ } \\
\cline { 2 - 10 } & $\delta_{L}$ & $\eta_{L}$ & $\delta_{L}$ & $\eta_{L}$ & $\delta_{L}$ & $\eta_{L}$ & $\delta_{L}$ & $\eta_{L}$ \\
\hline 0 & 70 & 0.22 & 74 & 0.22 & 64 & 0.21 & 69 & 0.16 \\
\hline 1 & 65 & 0.23 & 68 & 0.22 & 65 & 0.18 & 54 & 0.18 \\
\hline 2 & 50 & 0.24 & 55 & 0.22 & 47 & 0.22 & 48 & 0.21 \\
\hline 3 & 32 & 0.22 & 53 & 0.23 & 38 & 0.22 & 31 & 0.22 \\
\hline 4 & 11 & 0.21 & 12 & 0.23 & 7 & 0.22 & 9 & 0.24 \\
\hline 5 & -20 & 0.24 & -19 & 0.24 & -17 & 0.25 & -19 & 0.23 \\
\hline 6 & -62 & 0.23 & -58 & 0.22 & -65 & 0.18 & -58 & 0.20 \\
\hline 7 & 71 & 0.23 & 72 & 0.19 & 66 & 0.19 & 66 & 0.21 \\
\hline 8 & 13 & 0.30 & 12 & 0.31 & 9 & 0.33 & 11 & 0.33 \\
\hline 9 & -47 & 0.41 & -46 & 0.42 & -48 & 0.42 & -45 & 0.42 \\
\hline 10 & 72 & 0.57 & 72 & 0.57 & 72 & 0.55 & 70 & 0.53 \\
\hline 11 & 35 & 0.77 & 34 & 0.75 & 35 & 0.77 & 34 & 0.74 \\
\hline 12 & 17 & 0.89 & 17 & 0.88 & 18 & 0.89 & 17 & 0.89 \\
\hline 13 & 9 & 0.95 & 9 & 0.94 & 9 & 0.94 & 8 & 0.94 \\
\hline 14 & 4.5 & 0.98 & 4 & 0.98 & 4 & 0.98 & 4 & 0.97 \\
\hline 15 & 2 & 0.99 & & & & & 2 & 0.99 \\
\hline
\end{tabular}

Как следует из таблицы, результаты разных методов находятся в разумном согласии друг с другом. При этом наши результаты наиболее близки к результатам MKЭ.

Мы вычислили также поведение дифференциального сечения упругого $(d-A)$ рассеяния как функцию угла рассеяния на основе результатов разных методов, которые обсуждались выше. Для сечения использовалась следующая формула:

$$
\frac{d \sigma}{d \Omega}=\left|\frac{1}{2 i K_{j_{0}}} \sum_{L=0}^{L_{\max }}(2 L+1)\left(1-S_{L}\right) P_{L}(\cos \theta)\right|^{2},
$$

где $P_{L}(x)$ - полиномы Лежандра, $K_{j_{0}}$ - волновое число, соответствующее энергии центра масс падающего дейтрона, $S_{L}$ - парциальные элементы $S$-матрицы. В расчетах использовано значение $L_{\max }=15$.

Интересно также сравнить полученные сечения с сечениями, отвечающими рассеянию дейтрона как целого (т.е. без учета его внутреннего возбуждения) на потенциале свертки $V_{0}(\eta)$, определяемом как усреднение оператора внешнего взаимодей- 


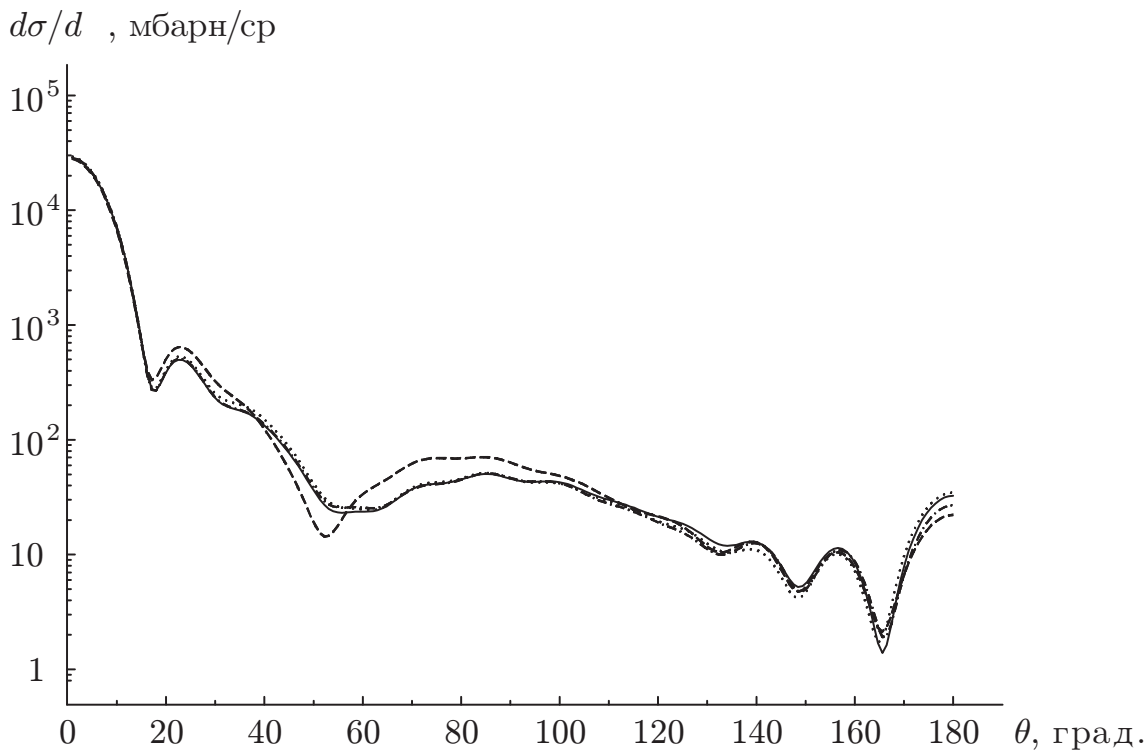

Рис. 1. Дифференциальные сечения упругого рассеяния дейтрона на ядре, полученные на основе МПДК при разных размерностях базиса свободных волновых пакетов: $N_{\mathrm{int}}=30$ (сплошная кривая), $N_{\mathrm{int}}=50$ (пунктирная кривая), $N_{\text {int }}=70,100$ (штрих-пунктирная кривая). Сечение для потенциала свертки обозначено штриховой кривой.

ствия $U_{1}$ по точной функции дейтрона $\left|Z_{0}\right\rangle$,

$$
V_{0}(\eta)=\frac{1}{4 \pi} \int d \boldsymbol{\xi} Z_{0}(\xi)\left[V_{2}\left(\left|\frac{1}{2} \boldsymbol{\xi}+\boldsymbol{\eta}\right|\right)+V_{3}\left(\left|\frac{1}{2} \boldsymbol{\xi}-\boldsymbol{\eta}\right|\right)\right] Z_{0}(\xi) .
$$

На рис. 1 приведена угловая зависимость дифференциального сечения рассеяния при $E_{d}=22.9$ МэВ, найденного на основе МПДК для базисов разной размерности. Для сравнения на графике приведены также сечения, рассчитанные для двухчастичной системы с потенциалом свертки. Снова видно, что размерность базиса $N=30$ вполне достаточна для расчетов. Кривые для $N_{\text {int }}=70$ и $N_{\text {int }}=100$ визуально неотличимы.

На рис. 2 приведены графики дифференциальных сечений, найденные на основе МПДК, CDCC-метода и МKЭ. Как видно из рисунка, небольшой разброс в значениях фазовых сдвигов, полученных в разных подходах, не приводит к существенным отличиям в угловых распределениях, причем максимальные отличия наблюдаются при больших углах.

Итак, показано, что развиваемый нами подход позволяет находить наблюдаемые в трехчастичной задаче рассеяния (например, составной частицы на ядре или электрона на водородоподобном атоме) на основе удобных аналитических конечномерных аппроксимаций канальной и полной резольвент.

Такое решение является намного более последовательным, чем традиционно используемые методы, поскольку позволяет избежать явного учета граничных условий. Это особенно важно для задач, в которых необходимо учитывать большое 


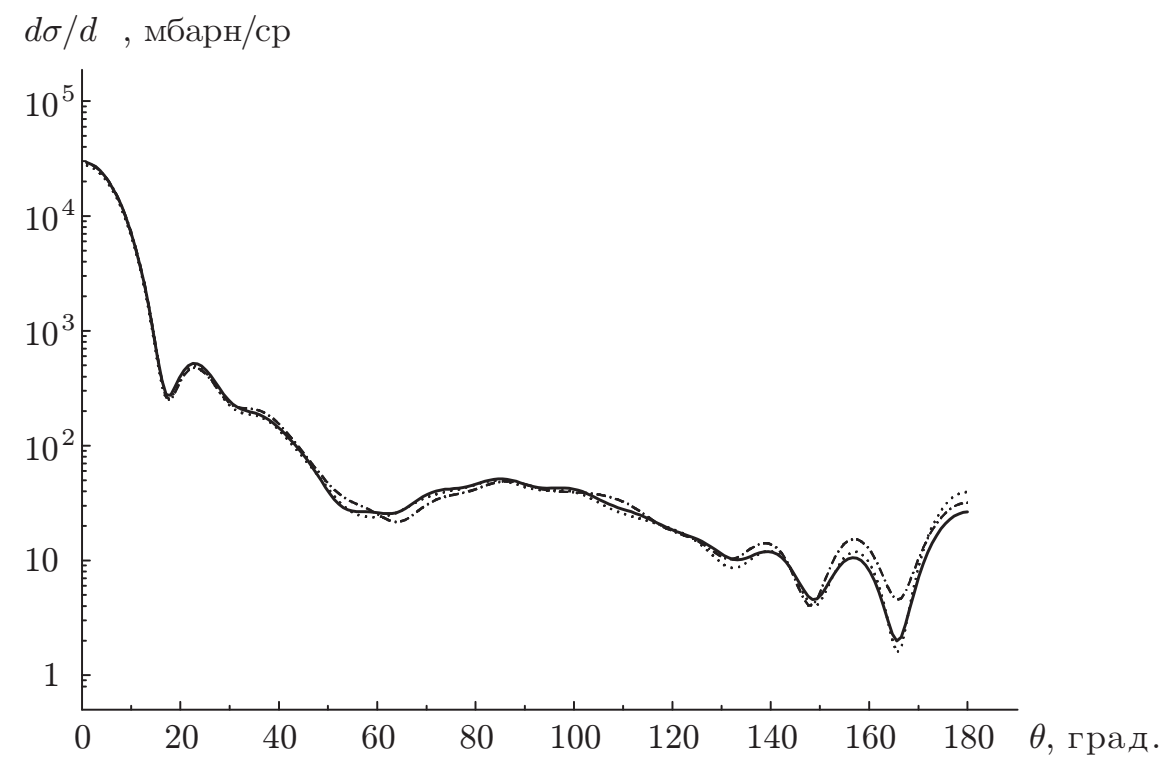

Рис. 2. Дифференциальные сечения упругого рассеяния дейтрона на ядре, полученные на основе трех методов: МПДК (сплошная кривая), МКЭ (пунктирная кривая) и CDCC-метода (штрих-пунктирная кривая).

количество парциальных волн в двухчастичных подсистемах. В этом случае решение системы уравнений связи каналов в CDCC-методе [9]-[11] становится очень громозким. В нашем методе при этом увеличивается лишь размерность используемых матриц, а сама схема решения остается прежней.

Подчеркнем, что МПДК не является лишь некоторым обобщением CDCC-метода, и область применимости МПДК не ограничивается решением рассмотренной упрощенной трехчастичной задачи. Полученые конечномерные представления для канальных резольвент позволяют использовать этот метод и в полной трехчастичной задаче с учетом граничных условий во всех асимптотических каналах.

\section{4. РАССЕЯНИЕ ТРЕХ ТОЖДЕСТВЕННЫХ ЧАСТИЦ}

В качестве еще одного примера использования пакетной техники в трехчастичной задаче рассмотрим решение уравнений Фаддеева для системы трех тождественных бозонов. Согласно [21] фаддеевские компоненты волновой функции такой системы связаны преобразованиями симметрии. Поэтому полная волновая функция может быть записана в следующем виде:

$$
\Psi=\left(1+P_{12} P_{23}+P_{13} P_{23}\right) \psi \equiv(1+P) \psi_{1},
$$

где $P_{i j}$ - операторы перестановки соответствующих частиц, $\psi_{1}$ - одна из трех одинаковых фаддеевских компонент волновой функции (отвечающая набору координат Якоби для канала 1). Будем считать, что в каждой двухчастичной подсистеме имеется одно связанное состояние, и рассмотрим процесс упругого рассеяния этого связанного состояния на третьей частице. 
Фаддеевская компонента $\psi_{1}$ полной волновой функции задачи рассеяния может быть найдена из интегрального уравнения Фаддеева

$$
\psi_{1}=\phi_{1}+G^{1} V_{1} P \psi_{1},
$$

где $\phi_{1}$ - начальная функция в канале $1, G^{1}$ - канальная резольвента, $V_{1}$ - оператор взаимодействия и $P$ - полный оператор перестановки частиц, определенный в (55). Численное решение этого уравнения представляет довольно сложную задачу, поскольку в правой части стоит та же неизвестная функция, что и в левой, но определенная в других (повернутых) координатах Якоби. Поэтому возникает необходимость включения в интегральное ядро матриц перехода из одного набора координат Якоби в другие, причем искомое решение в левой и правой частях уравнения (56) определяются на разных "сетках" координат, что приводит к необходимости выполнять на каждом шаге итераций этого уравнения (при его прямом численном решении) огромное количество многомерных интерполяций. Эти численные интерполяции резко замедляют весь процесс вычислений. Покажем, что при использовании пакетного формализма подобные сложности не возникают.

Снова введем собственный пакетный базис канала 1 и обозначим набор его функций через $\left\{\left|S^{(1)}\right\rangle\right\}$. Волновой функции начального состояния $\left|\phi_{1}\right\rangle$ будет соответствовать состояние $\left|S_{0}\right\rangle=\left|Z_{0}, X_{j_{0}}\right\rangle$, которое является произведением волновой функции связанного состояния $\left|Z_{0}\right\rangle$ двухчастичного гамильтониана $h_{1}$ и свободного волнового пакета $\left|X_{j_{0}}\right\rangle$, отвечающего двухчастичному гамильтониану $h_{0}^{1}$. В силу тождественности частиц собственные пакетные базисы в других каналах будут отличаться только перестановкой частиц с переходом к другим наборам координат Якоби.

Применим далее к уравнению (56) пакетное приближение, т.е. разложим фаддеевскую компоненту волновой функции по собственному пакетному базису канала 1 ,

$$
\left|\psi_{1}\right\rangle=\sum_{S} O_{S}\left|S^{(1)}\right\rangle
$$

и заменим начальную функцию $\left|\phi_{1}\right\rangle$ соответствующим ей стационарным волновым пакетом. Далее представим канальную резольвенту в виде ее разложения по пакетому базису (30)-(35). В случае трех тождественных бозонов получим из (56) следующее уравнение:

$$
\sum_{S} O_{S}\left|S^{(1)}\right\rangle=\frac{\left|S_{0}^{(1)}\right\rangle}{\sqrt{\Delta_{j}}}+2 \sum_{S, S^{\prime}}\left[G^{1}(E)\right]_{S}\left|S^{(1)}\right\rangle\left\langle S^{(1)}\left|V_{1}\right| S^{\prime(2)}\right\rangle O_{S^{\prime}} .
$$

Здесь мы учли свойство симметрии, согласно которому $\left\langle S^{(1)}\left|V_{1}\right| S^{\prime}(2)\right\rangle=$ $\left\langle S^{(1)}\left|V_{1}\right| S^{\prime(3)}\right\rangle$. Обозначим матричные элементы оператора взаимодействия между функциями пакетного базиса, определенными в разных наборах координат Якоби, как

$$
U_{S S^{\prime}} \equiv 2\left\langle S^{(1)}\left|V_{1}\right| S^{(2)}\right\rangle .
$$

Используя ортогональность функций пакетного базиса, получаем следующую систему линейных уравнений для коэффициентов $O_{S}$ :

$$
O_{S}=\frac{\delta_{S S_{0}}}{\sqrt{\Delta_{j_{0}}}}+\left[G^{1}(E)\right]_{S} \sum_{S^{\prime}} U_{S S^{\prime}} O_{S^{\prime}}
$$


решение которой записывается в виде

$$
O_{S}=\frac{\left[\mathbf{1}-\mathbf{G}^{1}(E) \mathbf{U}\right]_{S S_{0}}^{-1}}{\sqrt{\Delta_{j_{0}}}}, \quad E \in\left(\mathcal{E}_{j_{0}-1}+\varepsilon_{0}, \mathcal{E}_{j_{0}}+\varepsilon_{0}\right),
$$

где жирным шрифтом представлены матрицы соответствующих операторов в пакетном базисе, а $\varepsilon_{0}-$ энергия связи пары частиц.

Амплитуда упругого рассеяния согласно [21] определяется по известной формуле

$$
A_{\mathrm{el}}=\left\langle\phi_{1}\left|\bar{V}_{1}\right| \Psi\right\rangle=\left\langle\phi_{1}\left|V_{1} P\right| \psi_{1}\right\rangle,
$$

где $\bar{V}_{1}$ - оператор "внешнего" взаимодействия в канале 1. Заменим далее в правой части функцию начального состояния $\phi_{1}$ и функцию $P\left|\psi_{1}\right\rangle$ их "пакетными" представлениями, в результате получим следующее приближенное выражение для амплитуды упругого рассеяния:

$$
\begin{gathered}
A_{\mathrm{el}}(E) \approx \frac{2 \sum_{S} O_{S}\left\langle S_{0}^{(1)}\left|V_{1}\right| S^{(2)}\right\rangle}{\sqrt{\Delta_{j_{0}}}}=\frac{\left[\mathbf{U}\left(\mathbf{1}-\mathbf{G}^{1}(E) \mathbf{U}\right)^{-1}\right]_{S_{0} S_{0}}}{\Delta_{j_{0}}}, \\
E \in\left(\mathcal{E}_{j_{0}-1}+\varepsilon_{0}, \mathcal{E}_{j_{0}}+\varepsilon_{0}\right) .
\end{gathered}
$$

Эта формула аналогична формуле (41) для амплитуды упругого рассеяния составной частицы в поле ядра. Отличие состоит лишь в матрице эффективного потенциала взаимодействия. В данном случае эта матрица несимметрична, т.е. неэрмитова. Следует отметить, что при использовании удобных $L_{2}$-базисов для аппроксимации пакетных базисов матричные элементы потенциала (58) также могут быть найдены в аналитическом виде.

В качестве численной иллюстрации рассмотрим квартетное $(n-d)$-рассеяние с простым $s$-волновым локальным потенциалом Мальфлие-Тьона I-III

$$
V_{1}(\xi)=V_{R} \frac{e^{-\mu_{R} \xi}}{\xi}-V_{A} \frac{e^{-\mu_{A} \xi}}{\xi},
$$

где

$$
\begin{array}{rlrl}
V_{R} & =1438.72 \mathrm{MэB} \cdot \Phi_{\mathrm{M},} & & \mu_{R}=3.11 \Phi_{\mathrm{M}}{ }^{-1}, \\
V_{A} & =626.885 \mathrm{MэB} \cdot \Phi_{\mathrm{M},}, & \mu_{A}=1.55 \Phi_{\mathrm{M}}{ }^{-1} .
\end{array}
$$

Амплитуда упругого рассеяния в рассматриваемой системе определяется тем же соотношением (61), что и в случае трех бозонов, только в определении матричных элементов оператора взаимодействия (58) множитель 2 следует заменить на -1 , что связано с перекрыванием спиновых частей волновых функций в разных наборах координат Якоби (см. [2]).

При построении пакетного базиса применялись псевдосостояния двухчастичных гамильтонианов $h_{1}$ и $h_{0}^{1}$, полученные их диагонализацией на гауссовом базисе:

$$
Z_{i}(\xi) \approx \sum_{k=1}^{K} C_{i}^{k} e^{-\alpha_{k} \xi^{2}}, \quad X_{j} \approx \sum_{n=1}^{N} D_{j}^{n} e^{-\beta_{n} \eta^{2}}
$$

где для задания сетки масштабных параметров $\alpha_{k}$ и $\beta_{k}$ гауссова базиса использовалась геометрическая прогрессия:

$$
\alpha_{k}=\alpha_{0} \cdot a^{k-1}, \quad k=1, \ldots, K ; \quad \beta_{n}=\beta_{0} \cdot b^{n-1}, \quad n=1, \ldots, N .
$$


$\delta$, град.

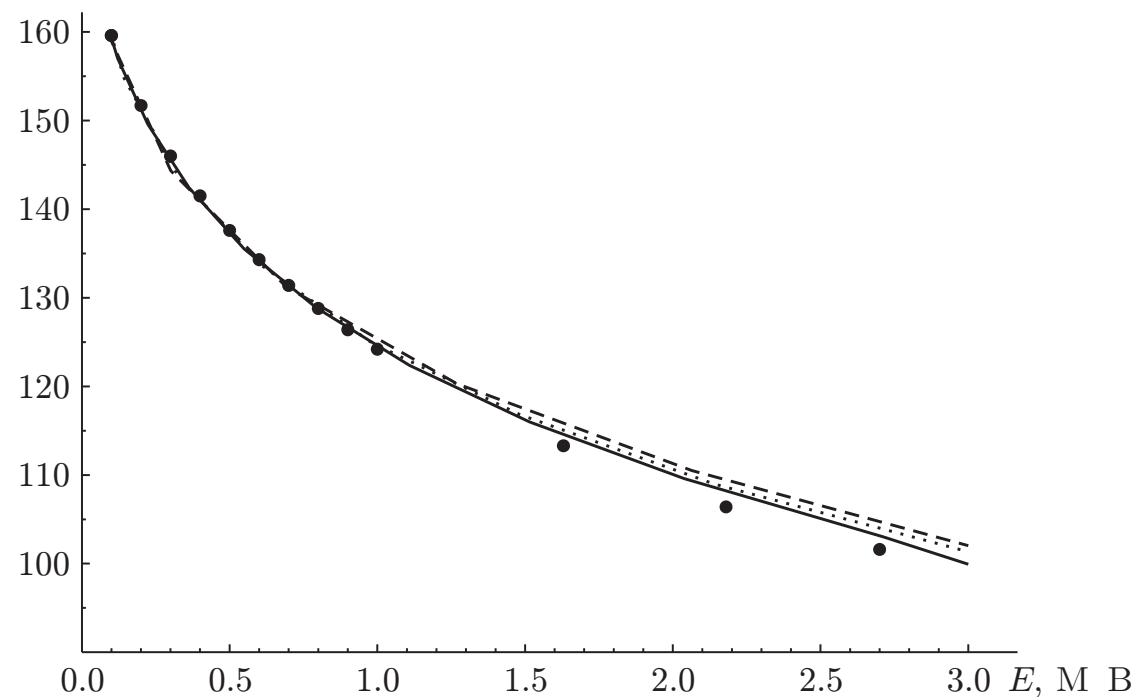

Рис. 3. Фазовые сдвиги упругого $s$-волнового $(n-d)$-рассеяния в квартетном канале, найденные на основе МПДК для различных размерностей базиса: размерности $35 \times 35$ отвечает штриховая линия, размерности $20 \times 41$ - пунктирная линия, размерности $20 \times 49$ - сплошная линия. Точками обозначен результат прямого численного решения уравнения Фаддеева [22].

При таком выборе базиса матричные элементы потенциала (58) полностью вычисляются аналитически.

На рис. 3 представлен $s$-волновой фазовый сдвиг квартетного упругого нейтрондейтронного рассеяния как функция энергии, рассчитанный нами на основе МПДК. Для сравнения на рисунке приведены также фазовые сдвиги, найденные на основе прямого численного решения уравнения Фаддеева в конфигурационном пространстве [22]. Согласие наших результатов с результатами прямого решения уравнения Фаддеева показывает, что пакетная дискретизация континуума хорошо работает и в полной трехчастичной задаче.

\section{5. ЗАКЛЮЧЕНИЕ}

Выполнено трехчастичное обобщение предложенного в наших предыдущих работах [17], [18] метода пакетной дискретизации континуума. Подробно изучены свойства трехчастичных стационарных волновых пакетов, которые строятся в каждом наборе координат Якоби трехчастичной системы. Получены в явном виде конечномерные представления для канальных резольвент в собственных пакетных базисах. Показано, что, как и в двухчастичном случае, эти спектральные представления удобно использовать при решении интегральных уравнений для волновых функций рассеяния и операторов системы. Эффективность подхода продемонстрирована для двух типов трехчастичных задач: рассеяние составной частицы на ядре-мишени и 
рассеяние в системе трех тождественных частиц. Полученные результаты хорошо согласуются с результатами других методов.

Из предложенных ранее подходов, использующих дискретные базисы для решения задач рассеяния нескольких тел, наиболее близкими к развитому в этой работе являются два. Первый из них - это метод связанных каналов дискретизованного континуума [9]-[11], который основан на дискретизации спектра только в одной двухчастичной подсистеме трехчастичной системы. Такое приближение сводит поиск наблюдаемых к решению громоздкой системы связанных дифференциальных уравнений для компонент волновой функции системы. В нашем подходе дискретизации проводится полностью - дискретизуются трехчастичные спектры канальных гамильтонианов. Это, во-первых, позволяет использовать интегральные уравнения для нахождения наблюдаемых, что значительно упрощает вычислительную схему; во-вторых, открывает возможность неявного учета граничных условий во всех асимптотических каналах трехчастичной системы.

Другой близкий по духу подход, предложенный для решения интегральных уравнений Меркурьева-Фаддеева в задаче рассеяния трех заряженных частиц [15], использует конечномерное представление трехчастичной кулоновской резольвенты в так называемом базисе Штурма-Кулона. При этом фаддеевские компоненты волновых функций системы представляются в виде разложений по базисам указанного типа для каждого набора координат Якоби. Хотя формальная схема конечномерного проектирования ядер интегральных уравнений, развиваемая в этих работах близка к нашей, в целом наш метод имеет существенные отличия. Прежде всего, они связаны с тем, что в МПДК проекционная схема удачно сочетается с формализмом стационарных волновых пакетов. В частности, полученные явные выражения для конечномерных пакетных проекций канальных резольвент в сочетании с использованием универсального базиса гауссова типа [17] позволяют (почти во всех случаях) полностью аналитически вычислить ядра и неоднородные члены используемых трехчастичных уравнений. Кроме того, сама идеология стационарных волновых пакетов (как некоторого усреднения точных решений по энергетическим бинам) должна хорошо работать в тех случаях, которые вызывают большие сложности при точной трактовке, например, при решении кулоновской задачи (как для притягивающего, так и для отталкивающего кулоновского взаимодействия) вблизи двух- и трехчастичного порога [17]. В этом случае усреднение по энергии позволяет существенно сгладить соответствующие кулоновские сингулярности в ядрах уравнений и оперировать при решении с достаточно гладкими по энергии функциями. И, наконец, легко просматривается обобщение нашего подхода на задачи рассеяния четырех и большего числа частиц. Авторы надеются в дальнейшем развить данную технику для решения кулоновской задачи трех тел выше трехчастичного порога и сравнить оба метода более предметно.

В целом следует ожидать, что развитие пакетной техники может привести к открытию нового пути решения задач многочастичного рассеяния, ведущего не только к кардинальному упрощению соответствующих расчетов, но и к развитию нового языка для описания процессов рассеяния полностью в дискретном представлении. При этом вместо непрерывной зависимости от энергии всех операторов теории рассеяния появляются их дискретные (по энергии) матричные аналоги. Эта дискретность не только более точно соответствует экспериментам по рассеянию частиц (где реги- 
стрирующая аппаратура всегда имеет конечное энергетическое разрешение, часто не такое уж малое), но и дает возможность эффективно регуляризовать нежелательное сингулярное энергетическое поведение многочастичных операторов теории рассеяния (например, вблизи соответствующих порогов) путем перехода к пакетному представлению, что является по сути некоторым энергетическим усреднением. Будущие исследования покажут, насколько эти ожидания являются оправданными.

Благодарности. Работа выполнена при частичной поддержке РФФИ (гранты № 04-02-16602, 05-02-17407, 05-02-04000).

\section{Список литературы}

[1] Л. Д. Фаддеев, ЖЭТФ, 39 (1960), 1459; С. П. Меркурьев, Л. Д. Фаддеев, Квантовая теория рассеяния для систем нескольких частии, Наука, М., 1985.

[2] Э. Шмид, Х. Цигельман, Проблема трех тел в квантовой механике, Наука, М., 1979.

[3] C. Gignoux, A. Laverne, Phys. Rev. Lett., 29 (1972), 436; N. W. Schellingerhout, "Numerical methods in configuration-space $A=3,4$ bound-state and scattering calculations", Few-Body Problems in Physics'93, Proc. XIVth European Conf. on Few-Body Problems in Physics (Amsterdam, Netherlands, 1993), eds. B. L. G. Bakker, R. van Dantzig, Springer, Wien, 1994, 361; V. M. Suslov, B. Vlahovic, Phys. Rev. C, 69 (2004), 044003; F. Ciesielski, J. Carbonell, Phys. Rev. C, 58 (1998), 58.

[4] W. Glöckle, H. Witała, D. Huber et al., Phys. Rep., 274 (1996), 107.

[5] R. Lazauskas, J. Carbonell, Phys. Rev. C, 70 (2004), 044002.

[6] W. Shadow, Ch. Elster, W. Glöckle, Three-body scattering below breakup threshold: an approach without using partial waves, nucl-th/9903020.

[7] Н. А. Yamani, M. S. Abdelmonem, J. Phys. B, 30 (1997), 1633; С. А. Зайцев, Ю. Ф. Смирнов, А. М. Широков, ТМФ, 117 (1998), 227.

[8] J. J. Bevelacqua, R. J. Philpott, Nucl. Phys. A, 275 (1977), 301.

[9] N. Austern, C. M. Vincent, J. P. Farrell, Ann. Phys., 96 (1976), 333; 114 (1978), 93.

[10] H. Amakawa, A. Mori, H. Nishioka et al., Phys. Rev. C, 23 (1981), 583.

[11] R. Y. Rasoanaivo, G.H. Rawitscher, Phys. Rev. C, 39 (1989), 1709; T. Matsumoto, T. Kamizato, K. Ogata et al., Phys. Rev. C, 68 (2003), 064607.

[12] J. R. Winick, W.P. Reinhardt, Phys. Rev. A, 18 (1978), 910; 925.

[13] M. Čižek, J. Horaček, H.-D. Meyer, Comput. Phys. Commun., 131 (2000), 41.

[14] V.D. Efros, W. Leidemann, G. Orlandini, Phys. Rev. C, 58 (1998), 582.

[15] Z. Papp, W. Plessas, Phys. Rev. C, 54 (1996), 50; Z. Papp, S. L. Yakovlev, The three-body Coulomb scattering problem in discrete Hilbert-space basis representation, nucl-th/9903078; Z. Papp, C-.Y. Hu, Z. T. Hlousek et al., Phys. Rev. A, 63 (2001), 062721 .

[16] В. И. Кукулин, О. А. Рубцова, ТМФ, 130 (2002), 64; 134 (2003), 460; 139 (2004), 291.

[17] В. И. Кукулин, О. А. Рубцова, ТМФ, 145 (2005), 393.

[18] А. И. Базь, Я. Б. Зельдович, А. М. Переломов, Рассеяние, реакции и распады в нерелятивистской квантовой механике, Наука, М., 1971.

[19] R. Kozack, F. S. Levin, Phys. Rev. C, 36 (1987), 883.

[20] О. А. Рубцова, В. И. Кукулин, ЯФ, 64 (2001), 1769.

[21] W. Glöckle, The Quantum Mechanical Few-Body Problem, Texts Monogr. Phys., Springer, Berlin, 1983.

[22] C. R. Chen, G. L. Payne, J. L. Friar, B. F. Gibson, Phys. Rev. C, 39 (1989), 1261.

Поступила в редакцию 22.04.2005, после доработки 19.05.2006 